\title{
A Study of Perks-II Distribution via Bayesian Paradigm
}

\author{
A.K.Chaudhary (Ph D) \\ Associate Professor, Nepal Commerce Campus
}

\begin{abstract}
In this paper, the Markov chain Monte Carlo (MCMC) method is used to estimate the parameters of Perks-II distribution based on a complete sample. The procedures are developed to perform full Bayesian analysis of the Perks-Il distributions using Markov Chain Monte Carlo (MCMC) simulation method in OpenBUGS, established software for Bayesian analysis using Markov Chain Monte Carlo (MCMC) methods. We have obtained the Bayes estimates of the parameters, hazard and reliability functions, and their probability intervals are also presented. We have also discussed the issue of model compatibility for the given data set. A real data set is considered for illustration under gamma sets of priors.
\end{abstract}

Kewwords: Bayesian estimation, Maximum likelihood estimation, Markov chain Monte Carlo, Model validation, OpenBUGS, Perks-II distribution

\section{Introduction}

[Perks (1932)] proposed the four-parameter extension of the Gompertz-Makeham distribution that has hazard rate of the form

$$
h(x)=\frac{A+B e^{\lambda x}}{K e^{-\lambda x}+1+D e^{\lambda x}} ; x>0 .
$$

The choice $\mathrm{K}=\mathrm{D}=0$ yields the Gompertz-Makeham hazard rate. It appears that Perks intended the parameters to be nonnegative. [Marshall and Olkin (2007)] have shown that we can not take $\mathrm{D}=0$. However, Gompertz-Makeham distribution can be obtained by setting $\mathrm{K}=0$ and taking limit as $D \rightarrow 0$.

Recently, two modified versions of Perks distribution are appeared in literature. The first one has been introduced by [Richards (2008, 2012)] by modifying the hazard function of Perks distribution as

$$
h(x)=\frac{\alpha \beta \mathrm{e}^{\beta x}}{\left(1+\alpha \mathrm{e}^{\beta x}\right)} \quad ; \alpha>0, \beta>0, x>0 .
$$

Applications of the Perks distribution to actuarial science include: models for pensioner mortality data [Richards (2008)]; parametric mortality projection models [Haberman and Renshaw (2011)]. The moments for this distribution do not appear to 
be available in closed form, [Nadarajah and Bakar (2012)]. We shall denote it as Perks I distribution.

The second version (Perks II distribution) has been introduced by [Yee (2012)]. The hazard function of Perks II distribution is given by

$$
h(x)=\frac{\alpha \mathrm{e}^{\beta x}}{\left(1+\alpha \mathrm{e}^{\beta x}\right)} \quad ; \alpha>0, \beta>0, x>0 .
$$

It is to be noted that most of the cited literatures are confined to classical developments and any systematic development on Bayesian results are rarely seen for the Perks I and Perks II distributions. The importance of the Bayesian method is well known both in the context of reliability studies and otherwise. Among several advantages, the most important is the fact that the Bayesian methods are equally well applicable for small sample sizes and censored data problems; the two common features in reliability data analyses. Chaudhary and Kumar (2013) obtained the maximum likelihood and Bayes estmates for the parameters of Perks-II distribution by MCMC method.

\section{Perks-II distribution}

\section{$2.1 \quad$ Model Analysis}

Cumulative distribution function (cdf):

The distribution function of Perks -I distribution with two parameters is given by

$$
F(x ; \alpha, \beta)=1-\left(\frac{1+\alpha}{1+\alpha \mathrm{e}^{\beta x}}\right)^{1 / \beta} \quad ; \alpha>0, \beta>0, x \geq 0 .
$$

where $\alpha>0$ and $\beta>0$ are the parameters. The Perks-II distribution will be denoted by $\operatorname{PS} 2(\alpha, \beta)$.

\section{Probability density function (pdf):}

The probability density function is given by

$$
f(x ; \alpha, \beta)=\alpha \mathrm{e}^{\beta x} \frac{(1+\alpha)^{1 / \beta}}{\left(1+\alpha \mathrm{e}^{\beta x}\right)^{1+(1 / \beta)}} \quad ; \alpha>0, \beta>0, x \geq 0 .
$$

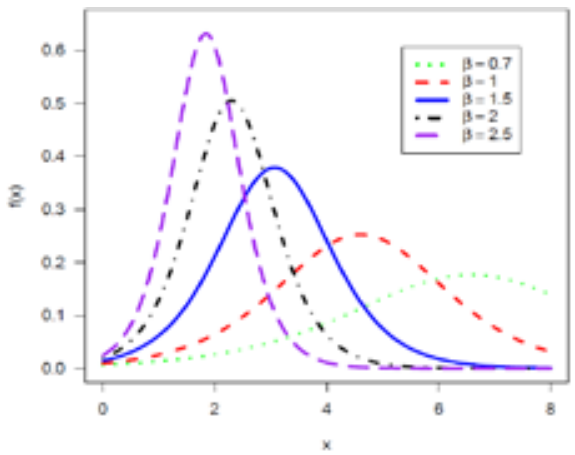

Fig 2.1 The probability density function of Perks I/ distribution for $\alpha=1$ and different values of $\beta$. 
The R functions dperks_II( ) and pperks_II( ) can be used for the computation of pdf and cdf respectively. Some of the typical PS2 density functions for different values of $\mathrm{b}$ and for $\mathrm{a}=1$ are depicted in Figure 2.1. It is clear from the Figure 2.1 that the density function of the $\operatorname{PS} 2(\alpha, \beta)$ distribution can take different shapes.

Mode:

Mode $=\frac{1}{\beta} \log \left(\frac{\beta}{\alpha}\right) \quad ; \beta \geq \alpha$

The Reliability/Survival function(sf):

The reliability/survival function is

$$
R(x ; \alpha, \beta)=\left(\frac{1+\alpha}{1+\alpha \mathrm{e}^{\beta x}}\right)^{1 / \beta} ; \alpha>0, \beta>0, x \geq 0 . .
$$

The R function sperks_II( ) computes the reliability/ survival function.

The hazard rate function(hrf):

The hazard rate function is

$$
h(x ; \alpha, \beta)=\frac{\alpha \mathrm{e}^{\beta x}}{\left(1+\alpha \mathrm{e}^{\beta x}\right)} \quad ; \alpha>0, \beta>0, x \geq 0 .
$$

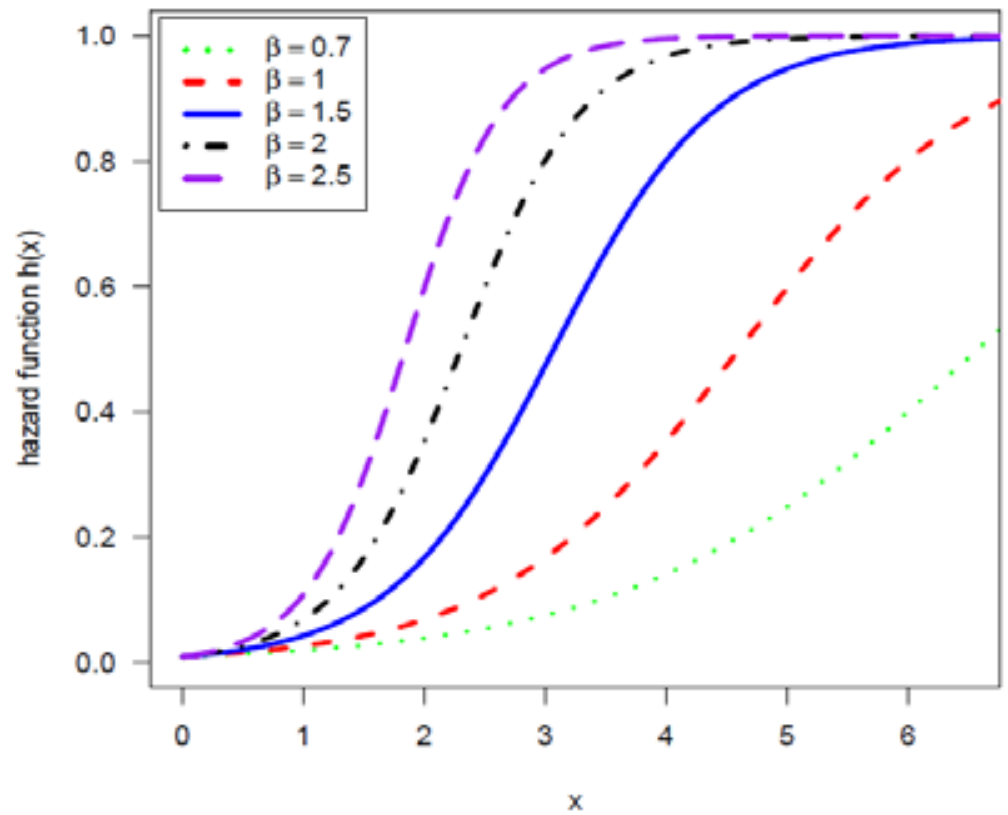

Fig 2.2 The hazard function of Perks II distribution for $\alpha=1$ and different values of $\beta$. 
Figure 2.2 exhibits the different hazard rate functions of PS2 $(\alpha, \beta)$ distribution. The associated $\mathrm{R}$ function hperks_II( ) computes the hazard rate function.

\section{The cumulative hazard function:}

The cumulative hazard function $\mathrm{H}(\mathrm{x})$ defined as

$$
\mathrm{H}(\mathrm{x})=-\log \{1-F(x)\}
$$

can be obtained with the help of pperks_II( ) function by choosing arguments lower .tail $=F A L S E$ and log. $p=T R U E$. i.e.

$$
\text { - pperks_II(x, alpha, beta, lower.tail=FALSE,log.p=TRUE) }
$$

\section{Failure rate average:}

Another relevant function useful in reliability analysis is failure rate average (FRA). The failure rate average of $\mathrm{X}$ is given by

$$
\operatorname{FRA}(\mathrm{x})=\frac{\mathrm{H}(\mathrm{x})}{\mathrm{x}}=\frac{1}{x} \int_{0}^{x} h(x) d x, \mathrm{x}>0,
$$

where $\mathrm{H}(\mathrm{x})$ is the cumulative hazard function. An analysis for $\mathrm{FRA}(\mathrm{x})$ on $\mathrm{x}$ permits to obtain the IFRA and DFRA classes. The R function hra.perks_II () can be used for the computation of FRA.

\section{The Quantile function:}

The quantile function is given by

$$
x_{p}=\frac{1}{\beta} \log \left[\frac{1}{\alpha}\left(\frac{(1+\alpha)}{(1-p)^{\beta}}-1\right)\right] ; 0<p<1 .
$$

For the computation of quantiles, the R function qperks_II() can be used.

\section{The random deviate generation:}

The random deviate can be generated from $\operatorname{PS} 2(\alpha, \beta)$ by

$$
x=\frac{1}{\beta} \log \left[\frac{1}{\alpha}\left(\frac{(1+\alpha)}{(1-u)^{\beta}}-1\right)\right] \quad ; 0<u<1 .
$$

where $\mathrm{u}$ has the $\mathrm{U}(0,1)$ distribution. The $\mathrm{R}$ function rperks_II( ) generates the random deviate from $\operatorname{PS} 2(\alpha, \beta)$.

For model choice purpose, the values of AIC and BIC can be computed using the $\mathrm{R}$ function abic.perksII( ) .

\section{Maximum Likelihood Estimation (MLE)}

For completeness purposes, in this section, we briefly discuss the maximum likelihood estimators (MLE's) of the two-parameter $\operatorname{PS} 2(\alpha, \beta)$ distribution.

Let $\mathrm{x}=(\mathrm{x} 1, \ldots, \mathrm{xn})$ be a random sample of size $\mathrm{n}$ from $\operatorname{PS} 2(\alpha, \beta)$, then the loglikelihood function $l(\alpha, \beta)$ can be written as; 


$$
l(\alpha, \beta)=n \log (\alpha)+\beta \sum_{i=1}^{n} x_{i}+\frac{n}{\beta} \log (1+\alpha)-\left(1+\frac{1}{\beta}\right) \sum_{i=1}^{n} \log \left\{1+\alpha \exp \left(\beta x_{i}\right)\right\}
$$

Therefore, to obtain the MLE's of $\alpha$ and $\beta$, we can maximize (3.4.10) directly with respect to $\alpha$ and $\beta$ or we can solve the following two non-linear equations using Newton-Raphson method.

$$
\begin{gathered}
\frac{\partial l}{\partial \alpha}=\frac{n}{\alpha}+\frac{n}{\beta(1+\alpha)}-\left(1+\frac{1}{\beta}\right) \sum_{i=1}^{n} \frac{\exp \left(\beta x_{i}\right)}{\left\{1+\alpha \exp \left(\beta x_{i}\right)\right\}}=0 \\
\frac{\partial l}{\partial \beta}=-\frac{n \log (1+\alpha)}{\beta^{2}}+\sum_{i=1}^{n} x_{i}+\frac{1}{\beta^{2}} \sum_{i=1}^{n} \log \left\{1+\alpha \exp \left(\beta x_{i}\right)\right\} \\
-\left(1+\frac{1}{\beta}\right) \sum_{i=1}^{n} \frac{\alpha x_{i} \exp \left(\beta x_{i}\right)}{\left\{1+\alpha \exp \left(\beta x_{i}\right)\right\}}=0
\end{gathered}
$$

\section{Bayesian Model Formulation}

The Bayesian model is constructed by specifying the prior distributions for the model parameters $\alpha$ and $\beta$, and then multiplying with the likelihood function to obtain the posterior distribution function.

Probability Model : $f(x \mid \alpha, \beta)$

Prior distribution : $p(\alpha, \beta)$

Data : $\underline{x}=\left(x_{1}, \ldots, x_{n}\right)$

Given a set of data $\underline{x}=\left(x_{1}, \ldots, x_{n}\right)$, the likelihood function is

$$
L(\alpha, \beta \mid \underline{x})=\alpha^{n}(1+\alpha)^{n / \beta} \exp \left(\beta \sum_{i=1}^{n} x_{i}\right)\left(\prod_{i=1}^{n}\left(1+\alpha e^{\beta x_{i}}\right)^{-(1+(1 / \beta))}\right)
$$

Denote the prior distribution of $\alpha$ and $\beta$ as $p(\alpha, \beta)$. The joint posterior is

$$
p(\alpha, \beta \mid \underline{x}) \propto L(\alpha, \beta \mid \underline{x}) p(\alpha, \beta)
$$

\section{Prior distributions:}

We assume the independent gamma priors for $\alpha \sim G\left(a_{1}, b_{1}\right)$ and $\beta \sim G\left(a_{2}, b_{2}\right)$ as

$$
\mathrm{p}(\alpha)=\frac{\mathrm{b}_{1}^{\mathrm{a}_{1}}}{\Gamma\left(\mathrm{a}_{1}\right)} \alpha^{\mathrm{a}_{1}-1} \mathrm{e}^{-\mathrm{b}_{1} \alpha} ; \alpha>0,\left(\mathrm{a}_{1}, \mathrm{~b}_{1}\right)>0
$$

and

$$
p(\beta)=\frac{b_{2}^{a_{2}}}{\Gamma\left(a_{2}\right)} \beta^{a_{2}-1} \mathrm{e}^{-b_{2} \beta} ; \beta>0,\left(a_{2}, b_{2}\right)>0
$$

\section{Posterior distribution:}

Combining the likelihood function with the prior via Bayes' theorem yields the posterior as 


$$
\begin{aligned}
p(\alpha, \beta \mid \underline{x})= & \alpha^{n}(1+\alpha)^{n / \beta} \exp \left(\beta \sum_{i=1}^{n} x_{i}\right)\left(\prod_{i=1}^{n}\left(1+\alpha e^{\beta x_{i}}\right)^{-(1+(1 / \beta))}\right) \\
& \left(\frac{b_{1}^{a_{1}}}{\Gamma\left(a_{1}\right)} e^{-b_{1} \alpha} \alpha^{a_{1}-1}\right)\left(\frac{b_{2}^{a_{2}}}{\Gamma\left(a_{2}\right)} e^{-b_{2} \beta} \beta^{a_{2}-1}\right)
\end{aligned}
$$

It can be written, upto proportionality, as

$$
p(\alpha, \beta \mid \underline{x}) \propto \alpha^{n+a_{1}-1}(1+\alpha)^{n / \beta} \beta^{a_{2}-1} \exp \left(-b_{1} \alpha-b_{2} \beta+\beta \sum_{i=1}^{n} x_{i}\right) T_{1}
$$

where $T_{1}=\prod_{i=1}^{n}\left(1+\alpha e^{\beta x_{i}}\right)^{-(1+(1 / \beta))}$

It can be observed that posterior distribution is complicated. We consider MCMC techniques for the Bayes analysis of the model .

\section{Gibbs Sampler : Algorithm}

For Gibbs sampler implementation, the full conditionals for $\alpha$ and $\beta$ upto proportionality can be specified as

(i) Full conditional distribution of the parameter $a$ for given $b$ and $x$

$$
p(\alpha \mid \beta, \underline{x}) \propto \alpha^{n+a_{1}-1}(1+\alpha)^{n / \beta} \exp \left(-b_{1} \alpha\right) T_{1}
$$

(ii) Full conditional distribution of the parameter $b$ for given $a$ and $x$

$$
p(\beta \mid \alpha, \underline{x}) \propto(1+\alpha)^{n / \beta} \beta^{a_{2}-1} \exp \left\{-\beta\left(b_{2}-\sum_{i=1}^{n} x_{i}\right)\right\} T_{1}
$$

We shall use OpenBUGS software to obtain posterior samples. As the Perks-II distribution is not available in OpenBUGS, it requires incorporation of a module in ReliaBUGS [Kumar et al.(2010)], subsystem of OpenBUGS for Perks-II.

A module dPerks2_T(alpha, beta) is written in Component Pascal for PerksII to perform full Bayesian analysis in OpenBUGS using the method described in [Thomas et al.(2006)], [Thomas(2010)], [Kumar et al.(2010)] and [Lunn et al.(2013)]. It is important to note that this module can be used for any set of suitable priors of the model parameters. Almost all aspects of the model in Bayesian framework can be studied using the developed module dPerks2_T(alpha, beta).

\section{Data analysis}

\section{Data Set :}

The following real data set is considered for illustration of the proposed methodology. The data below are from an accelerated life test of 59 conductors, failure times are in hours, and there are no censored observations, [Lawless (2003)].

2.997, 4.137, 4.288, 4.531, 4.700, 4.706, 5.009, 5.381, 5.434, 5.459, 5.589, 5.640, $5.807,5.923,6.033,6.071,6.087,6.129,6.352,6.369,6.476,6.492,6.515,6.522$, $6.538,6.545,6.573,6.725,6.869,6.923,6.948,6.956,6.958,7.024,7.224,7.365$, 7.398, 7.459, 7.489, 7.495, 7.496, 7.543, 7.683, 7.937, 7.945, 7.974, 8.120, 8.336, 8.532, 8.591, 8.687, 8.799, 9.218, 9.254, 9.289, 9.663, 10.092, 10.491, 11.038 


\section{Classical Analysis}

\subsection{Computation of MLE}

The maximum

likelihood estimates (MLEs) are obtained by direct maximization of the loglikelihood function $\ell(\alpha, \beta)$ - The advantage of this procedure is that it runs immediately using existing statistical packages such R $[\mathrm{R}$ Development Core Team, (2013)]. We consider the software $R$ through the Quasi-Newton algorithm [Lange (1999)] to compute the MLEs. The MLEs (and the corresponding standard errors in parentheses) and the maximized value of loglikelihood of the Perks II distribution parameters are given by

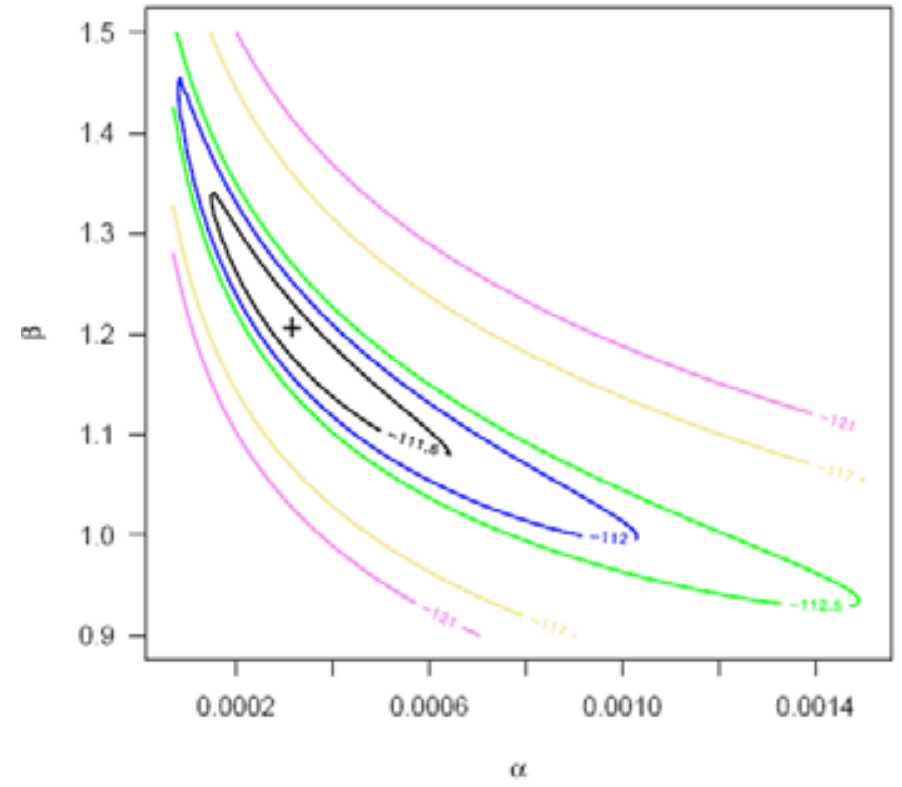

Fig 5.1 Contour plot

$$
\hat{\alpha}=0.0003158(0.0004036), \hat{\beta}=1.2041(0.22713) \text { and } \ell(\hat{\alpha}, \hat{\beta})=-111.398
$$

The contour plot in figure 5.1 computes the maximum likelihood estimates(MLEs) of the Perks-II distribution.

\subsection{Model Validation}

To check the validity of the model, we compute the Kolmogorov-Smirnov (KS) distance between the empirical distribution function and the fitted distribution function when the parameters are obtained by method of maximum likelihood.

For this, we can use $\mathrm{R}$ function ks.perks_II(). From Figure 5.2, it is clear that the estimated Perks II distribution provides excellent fit to the given

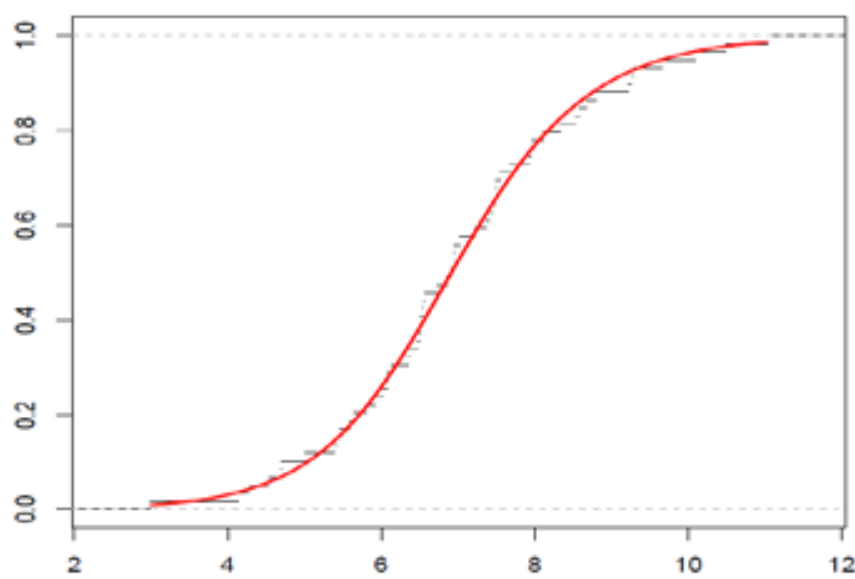

Fig 5.2 The graph of empirical and fitted distribution function. data. 
The following graphical methods are used for suitability of the model under consideration: (i) Quantile-Quantile(QQ) plot, and (ii)Probability -Probability(PP) plot
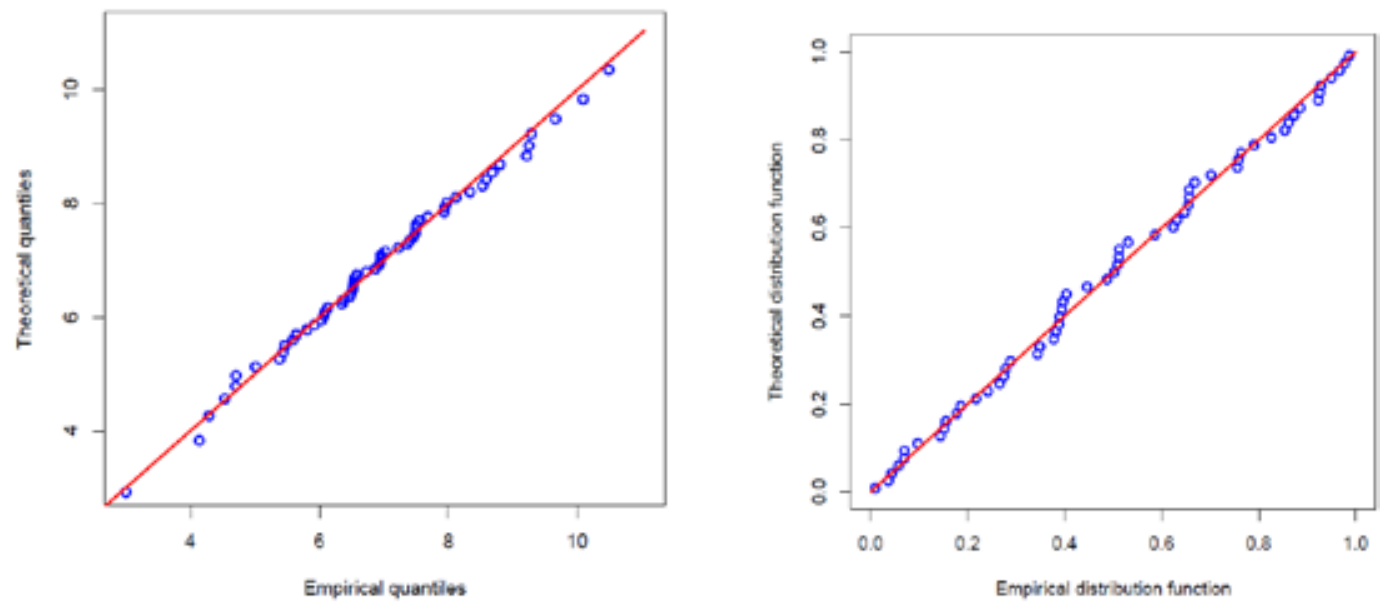

Fig 5.3 Quantile-Quantile(Q-Q) plot (left panel) and Probability-Probability(P-P) plot (right panel) using MLEs as estimate.

The corresponding $\mathrm{R}$ function qq.perks_II() for Q-Q plot and pp.perks_II() for P-P plot are used. As can be seen from the straight line pattern in Figure 5.3 the Perks II fits the data very well.

\section{Bayesian Analysis}

Script 3.2 : OpenBUGS script for the Bayesian analysis of Perks II distribution

model

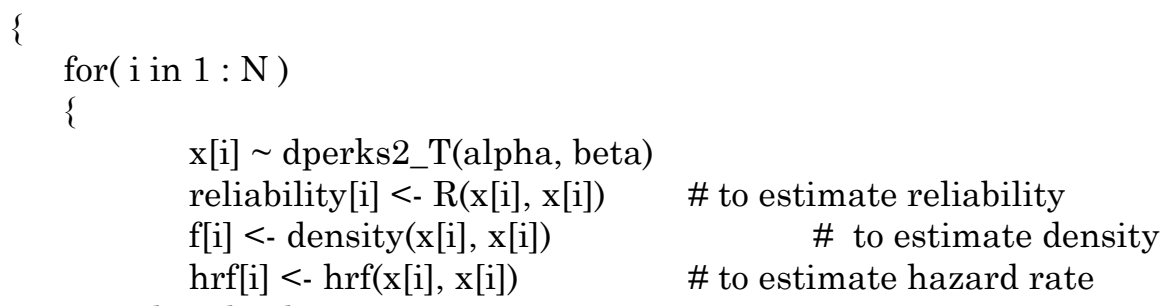

\section{DATA}

$\operatorname{list}(\mathrm{N}=59, \mathrm{x}=\mathrm{c}(2.997,4.137,4.288,4.531,4.700,4.706,5.009,5.381,5.434,5.459$, 
5.589, 5.640, 5.807, 5.923, 6.033, 6.071, 6.087, 6.129, 6.352, 6.369, 6.476, 6.492, $6.515,6.522,6.538,6.545,6.573,6.725,6.869,6.923,6.948,6.956,6.958,7.024$, 7.224, 7.365, 7.398, 7.459, 7.489, 7.495, 7.496, 7.543, 7.683, 7.937, 7.945, 7.974, 8.120, 8.336, 8.532, 8.591, 8.687, 8.799, 9.218, 9.254, 9.289, 9.663, 10.092, 10.491, 11.038))

\section{Init1}

$$
\text { list(alpha=0.001, beta=0.5) \# Chain } 1
$$

\section{Init2}

$$
\text { list(alpha=0.0001, beta }=1.0) \quad \text { \# Chain } 2
$$

We assume the independent gamma priors for $\alpha \sim G\left(a_{1}, b_{1}\right)$ and $\beta \sim G\left(a_{2}, b_{2}\right)$ with hyper parameter values $\mathbf{a}_{1}=\mathbf{b}_{1}=\mathbf{a}_{2}=\mathbf{b}_{2}=\mathbf{0 . 0 0 1}$. We run the model to generate two Markov Chains at the length of 40,000 with different starting points of the parameters. We have chosen initial values $(\alpha=0.001, \beta=0.5)$ for the first chain and $(\alpha=0.0001, \beta=1.0)$ for the second chain. The convergence is monitored using trace and ergodic mean plots. We find that the Markov Chain converge together after approximately 2000 observations. Therefore, burnin of 5000 samples is more than enough to erase the effect of starting point(initial values). Finally, samples of size 7000 are formed from the posterior by picking up equally spaced every fifth outcome (to minimize the auto correlation among the generated deviates.), i.e. thin $=5$, starting from 5001 .

Therefore, we have the posterior sample $\left(\alpha_{1}^{(j)}, \beta_{1}^{(j)}\right) ; \quad 1, \quad, 7000$ from chain 1 and $\left(\alpha_{2}^{(j)}, \beta_{2}^{(j)}\right) ; j=1, \ldots, 7000$ from chain 2 .

\subsection{Convergence diagnostics}

The sequential plot of parameters is the plot that most often exhibits difficulties in the Markov chain. Figure 5.4 shows the sequential realizations of the parameters of the model. In this case, Markov chain seems to be mixing well enough and is likely to be sampling from the stationary distribution.

The plot looks like a horizontal band, with no long upward or downward trends, then we have evidence that the chain has converged.

\section{Running Mean (Ergodic mean) Plot}

The running mean(ergodic average) is computed as the mean of all sampled values up to and including that at a given iteration. The convergence pattern based on ergodic average as shown in Figure 5.5 is obtained after generating a time series (iteration number) plot of the running mean for each parameter in the chain. The dotted line represents the mean of sample values. The plot shows that the ergodic mean stablizes as chain advances. 
Pravaha Journal-2018

\section{History(Trace) plot}
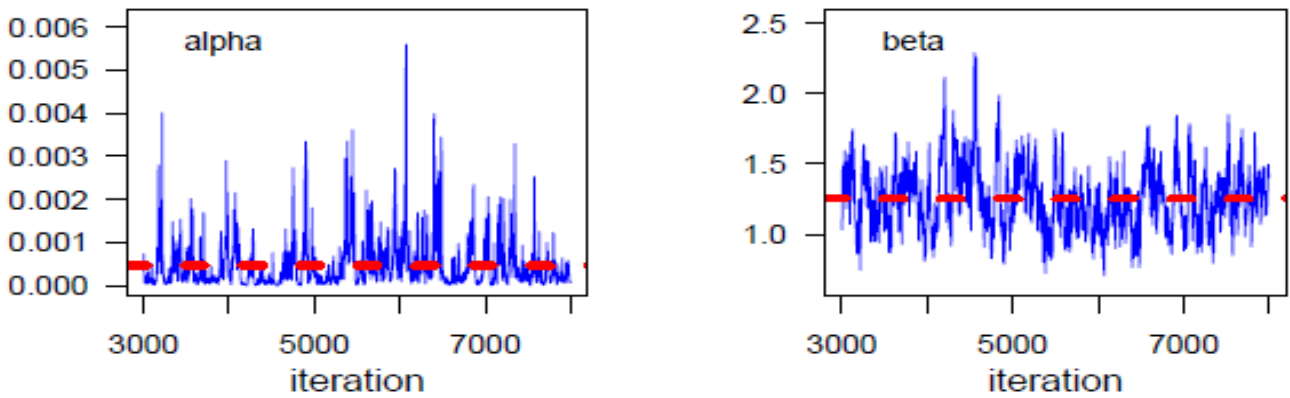

Fig 5.4 Sequential realization of the parameters $\alpha$ and $\beta$.
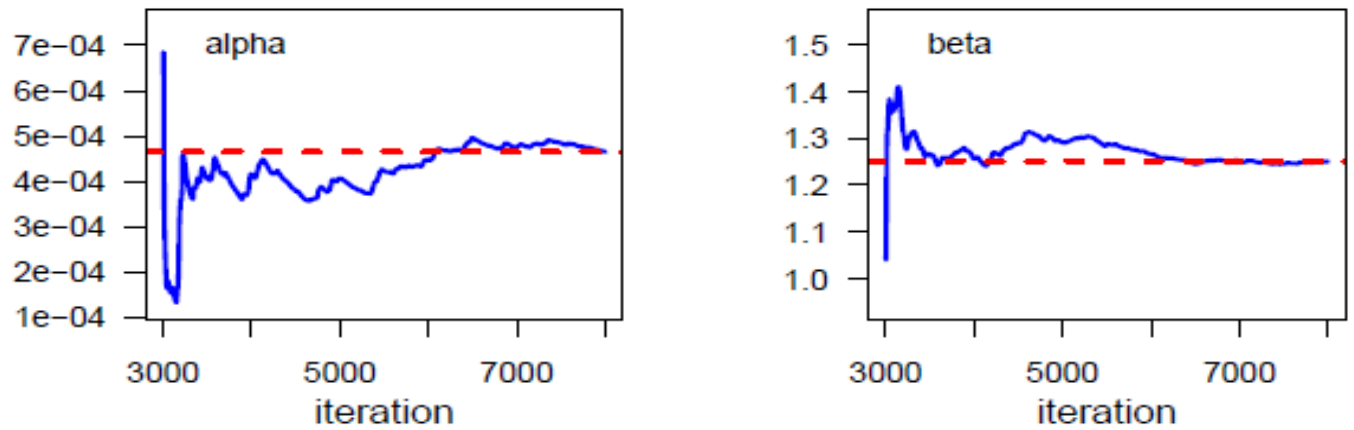

Fig. 5.5 The Ergodic mean plots for $\alpha$ and $\beta$.

\subsection{Posterior Analysis}

\section{(a) Numerical Summary}

In Table 5.1, we have considered various quantities of interest and their numerical values based on MCMC sample of posterior characteristics for Perks II distribution under gamma priors.

Table 5.1 : Numerical summaries based on MCMC sample of posterior characteristics for Perks II distribution under gamma priors

\begin{tabular}{lcccc}
\hline \multirow{2}{*}{ Characteristics } & \multicolumn{2}{c}{ Chain 1} & \multicolumn{2}{c}{ Chain 2} \\
\cline { 2 - 5 } & alpha & beta & alpha & beta \\
\hline Mean & 0.000453 & 1.259412 & 0.000528 & 1.218955 \\
Standard Deviation & 0.000580 & 0.224854 & 0.000624 & 0.211176 \\
Minimum & 0.000001 & 0.652100 & 0.000002 & 0.641700 \\
2.5th Percentile $\left(\mathrm{P}_{2.5}\right)$ & 0.000017 & 0.869790 & 0.000023 & 0.849598 \\
First Quartile $\left(\mathrm{Q}_{1}\right)$ & 0.000106 & 1.102000 & 0.000144 & 1.072000 \\
Median & 0.000255 & 1.244000 & 0.000318 & 1.204000 \\
Third Quartile $\left(\mathrm{Q}_{3}\right)$ & 0.000563 & 1.400000 & 0.000670 & 1.345000 \\
\hline
\end{tabular}


Pravaha Journal-2018

\begin{tabular}{lcccc}
\hline \multirow{2}{*}{\multicolumn{1}{c}{ Characteristics }} & \multicolumn{2}{c}{ Chain 1 } & \multicolumn{2}{c}{ Chain 2 } \\
\cline { 2 - 5 } & alpha & beta & alpha & beta \\
\hline 97.5th Percentile $\left(\mathrm{P}_{97.5}\right)$ & 0.002082 & 1.740025 & 0.002294 & 1.689025 \\
Maximum & 0.006530 & 2.365000 & 0.006848 & 2.156000 \\
Mode & 0.000085 & 1.240278 & 0.000118 & 1.164095 \\
Skewness & 3.329321 & 0.501056 & 2.974009 & 0.472158 \\
95\% Credible Interval & $(1.66 \mathrm{e}-05$, & $(0.8698,1.7400)$ & $(2.252 \mathrm{e}-05$, & $(0.84959$, \\
95\% HPD Credible & $0.0020)$ & & $0.00229)$ & $1.6890)$ \\
Interval & $(6.15 \mathrm{e}-07$, & $(0.8473,1.703)$ & $(2.154 \mathrm{e}-06$, & $(0.8063,1.626)$ \\
\hline
\end{tabular}

Highest probability density (HPD): The algorithm described by [Chen and Shao (1999)] is used to compute the HPD intervals under the assumption of unimodal marginal posterior distribution

\section{(b) Visual summary}

Box plots:

The boxplots of the parameters $\alpha$ and $\beta$ are displayed Figure 5.6.
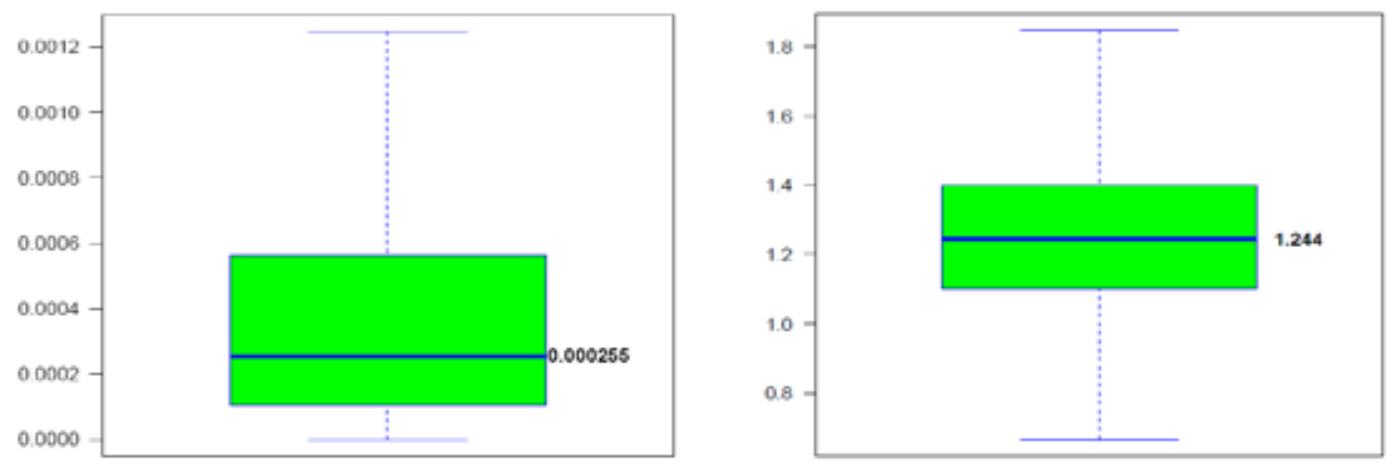

Fig 5.6 L eft panel :Boxplot for $\alpha$; Right panel : Boxplot for $\beta$.

\section{Kernel density estimates:}

The MCMC samples may be used to completely summarize the posterior uncertainty about the parameters $\alpha$ and $\beta$ through a kernel estimate of the posterior distribution. The marginal posterior density estimates of the parameters and their histograms based on samples of size 7000 are shown in Figure. 5.7 using the Gaussian kernel. 

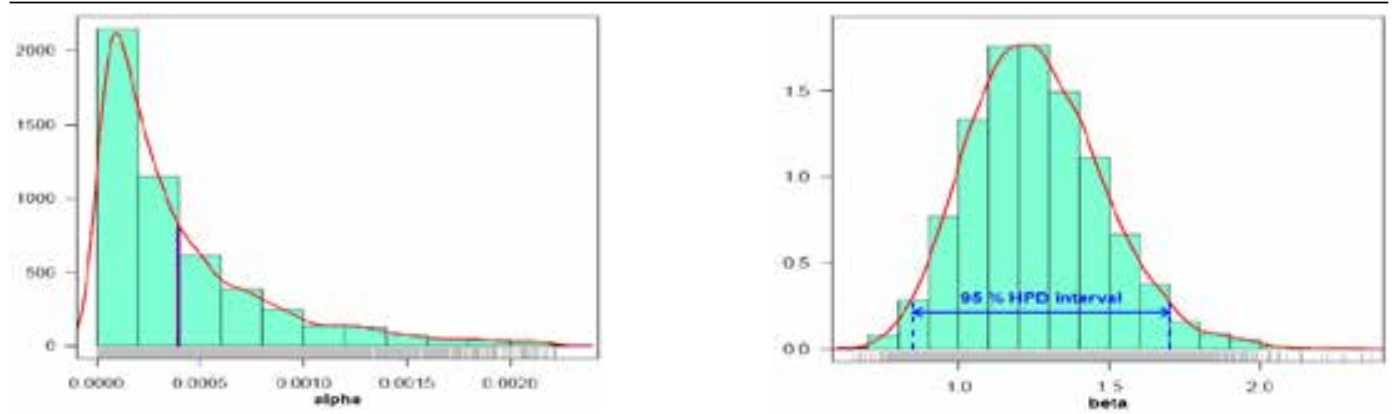

Fig 5.7 Kernel density estimate and HPD intervals for a (left panel) and b(right panel)

It is evident from the Figure 3.24 that the marginal distributions of $\beta$ is symmetrical whereas $\alpha$ shows positive skewness. The 95\% HPD interval for $\beta$ has been plotted in Figure 3.24 (right panel), whereas a vertical line is drawn at Bayes estimte (mean) of $\alpha$ (left panel).

\subsection{Comparison with MLE}

We have used graphical method for the comparison of Bayes estimates with $\mathrm{ML}$ estimates. In Figure 5.8, the density functions $\mathrm{f}(\mathrm{x} ; \hat{\alpha}, \hat{\beta})$ using MLEs and Bayesian estimates, computed via MCMC samples under gamma priors, are plotted. It is clear from the Figure 5.8 that the MLEs and the Bayes estimates with respect to the gamma priors are quite close and fit the data very well.

A further support for this finding can be obtained by inspecting the Figure 5.9. In Figure 5.9, we have plotted $2.5^{\text {th }}, 50^{\text {th }}$ and $97.5^{\text {th }}$ quantiles of the estimated density based on MCMC sample $\left(\alpha_{1}^{(j)}, \beta_{1}^{(j)}\right) ; j=1, \ldots, 7000$. Here the density is computed at each data point for 7000 posterior samples. The density corresponding to MLE has been plotted using the "plug-in" estimates of the parameters. It shows that we have a fairly good model for the given data set.

\subsection{Estimation of reliability function}

In this section, our main aim is to demonstrate the effectiveness of proposed methodology. For this, we have estimated

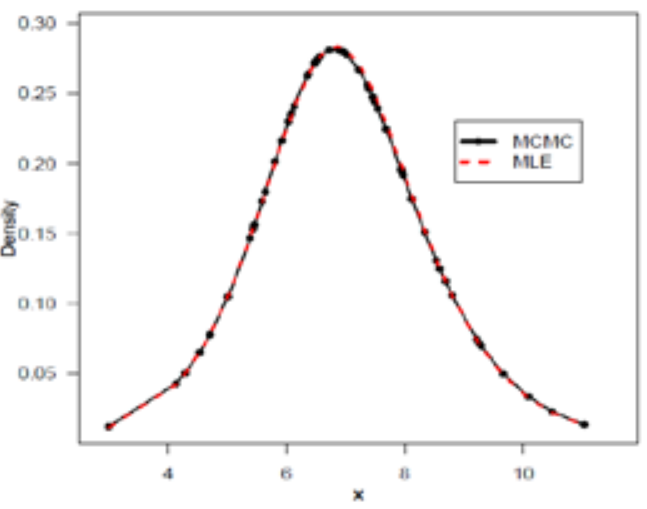

Fig 5.8 The density functions using $M L$ and Bayesian estimates

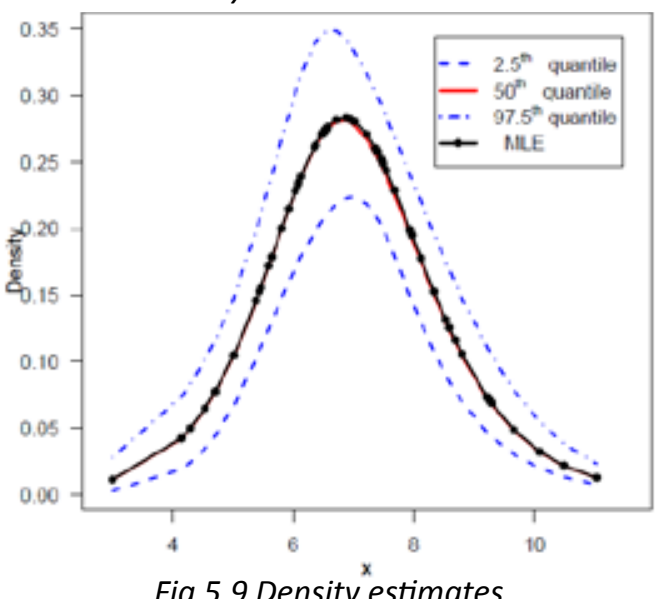

Fig 5.9 Density estimates the reliability function using MCMC samples under gamma priors. Since we have an 
effective MCMC technique, we can estimate any function of the parameters. We have used the Kaplan-Meier estimate of the reliability function to make the comparison more meaningful. The Figure 5.10, exhibits the estimated reliability function (dashed blue line: $2.5^{\text {th }}$ and $97.5^{\text {th }}$ quantiles; solid red line: $50^{\text {th }}$ quantile) using Bayes estimate based on MCMC output under independent gamma priors for both the parameters and the empirical reliability function (black solid line). The Figure 5.10 shows that reliability estimate based on MCMC is very closed to the empirical reliability estimates.

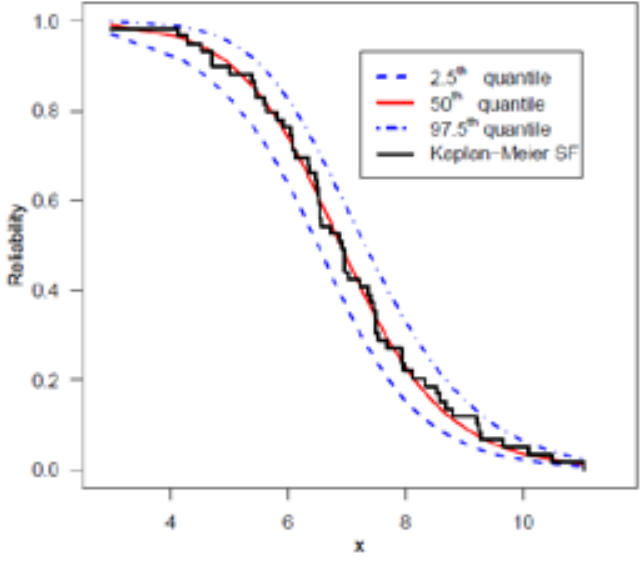

Fig 5.10 Reliability function estimate using MCMC and Kaplan-Meier estimate

\subsection{Estimation of Hazard and Reliability at $\mathbf{X}_{(7)}: \mathbf{t}=\mathbf{5 . 0 0 9}$}

The advantage of using the MCMC method over the MLE method is that we can always obtain a reasonable interval estimate of the parameters by constructing the probability intervals based on the empirical posterior distribution. This is often unavailable in maximum likelihood estimation. Indeed, the MCMC samples may be used to completely summarize the posterior uncertainty about the parameters $\alpha$ and $\beta$ through a kernel estimate of the posterior distribution. This is also true of any function of the parameters e.g. reliability and hazard functions. Suppose we wish to give point and interval estimates for reliability and hazard functions at the mission time $\mathrm{t}=5.009$ ( at the 7 th observed data point)..

The marginal posterior density estimates of the reliability and hazard functions and their histograms based on samples of size 7000 are shown in Figure. 5.11 and 5.12 using the Gaussian kernel. It is evident from the estimates that the marginal distribution of reliability is negatively skewed whereas hazard is positively skewed.

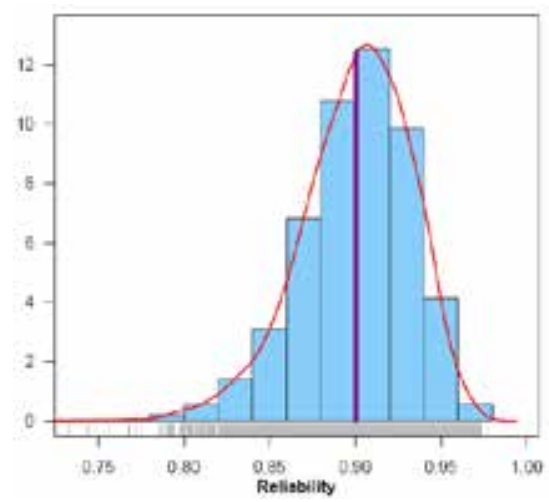

Fig 5.11 Visual summary of $\alpha$

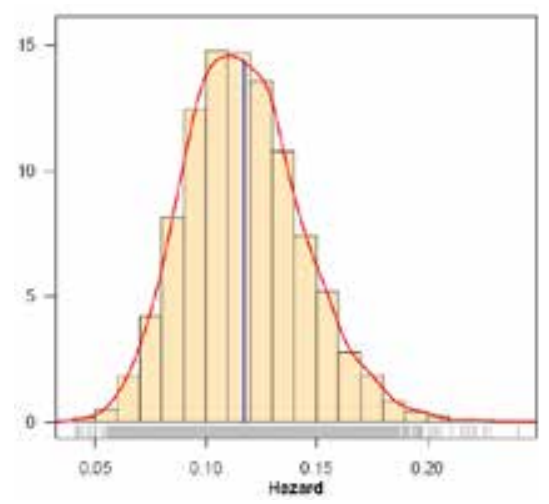

Fig 5.12 Visual summary of $\alpha$ 


\section{Pravaha Journal-2018}

The MCMC results of the posterior mean, median, mode, standard deviation(SD) and skewness of reliability and hazard functions are displayed in Table 5.2.The ML estimates of reliability and hazard function at $\mathrm{t}=5.009$ are computed using invariance property of the MLE.

Table 5.2 : Posterior summary of hazard and reliability

\begin{tabular}{lcccccc}
\hline \multirow{2}{*}{ Parameters } & \multirow{2}{*}{ MLE } & \multicolumn{5}{c}{ Based on MCMC output } \\
\cline { 3 - 7 } & & Mean & Median & Mode & SD & Skewness \\
\hline $\mathrm{h}(\mathrm{t}=5.009)$ & 0.1162 & 0.1173 & 0.1155 & 0.1113 & 0.0268 & 0.4304 \\
$\mathrm{R}(\mathrm{t}=5.009)$ & 0.9027 & 0.9003 & 0.9032 & 0.9069 & 0.0321 & -0.6168 \\
\hline
\end{tabular}

A trace plot is a plot of the iteration number against the value of the draw of the parameter at each iteration. Figure 5.13 display 7000 chain values for the reliability $\mathrm{R}(\mathrm{t}=5.009)$ and hazard $\mathrm{h}(\mathrm{t}=5.009)$ functions, with their sample median and $90 \%$ credible intervals.
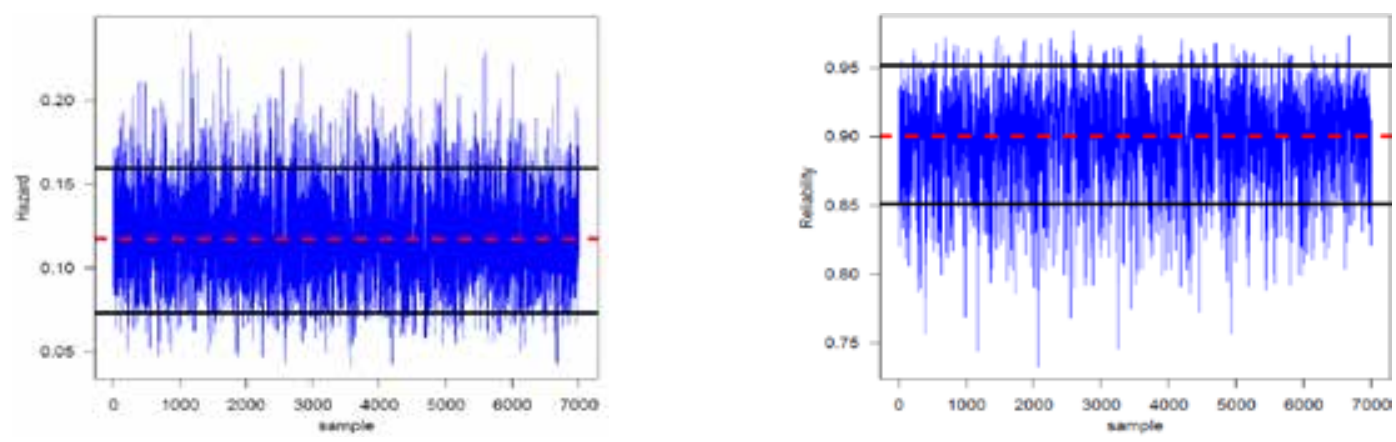

Fig 5.13 MCMC output of $h(t=5.009)$ and $R(t=5.009)$. Dashed line $(. .$.$) represents the posterior$ median and solid lines(-) represent lower and upper bounds of $90 \%$ probability intervals (HPD)

\subsection{Model compatibility}

\section{Posterior Predictive Checks:}

A natural way to assess the fit of a Bayesian model is to look at how well the predictions from the model agree with the observed data [Gelman (2003)] and [Gelman et al. (2004)]. We do this by comparing the posterior predictive simulations with the data.
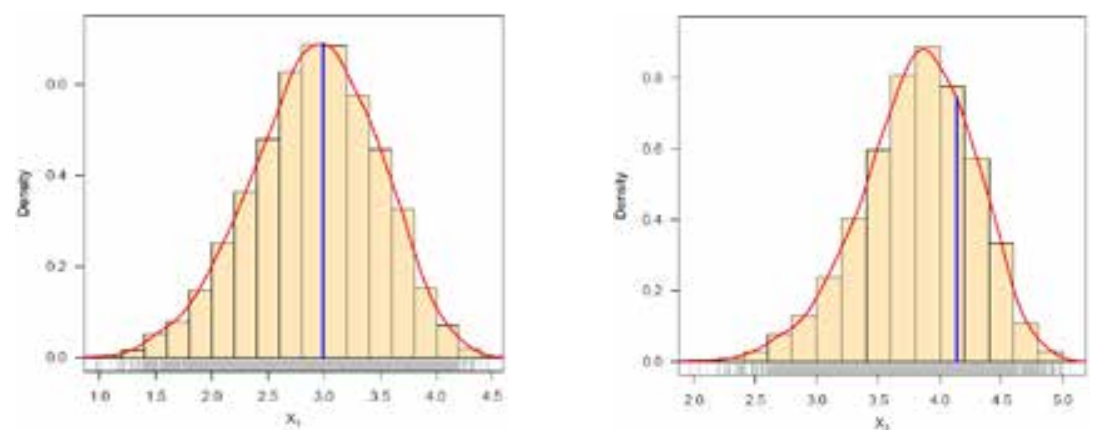

Fig 5.14 Density estimates of the two smallest order future observations, vertical lines represent corresponding observed values 
There are several approaches available for the study of model compatibility in Bayesian framework. Predictive simulation is an easiest and flexible one. The basic idea of studying the model compatibility through predictive simulation is to compare the observed data or some function of it with the data that would have been anticipated from the assumed model called the predictive data. If the two data sets compare favourably, the assumed model can be considered to be an appropriate choice for the data in hand, [Gupta et al. (2008)]. Modern Bayesian computational tools however provide straightforward solutions as one can easily simulate predictive samples if MCMC outputs are available from the posterior corresponding to the assumed model. Most of the standard numerical and graphical methods based on predictive distribution can then be easily implemented to study the compatibility of the model.

Comparison of empirical distribution function plots based on the observed and the predictive data may be considered as an informal way to check discrepancies between the data and the model.

To obtain further clarity on our conclusion for the study of model compatibility, we have considered plotting of density estimates of two smallest and two largest replicated future observations from the model with superimposed corresponding observed data. For this purpose, 5000 samples have been drawn from the posterior using MCMC procedure and then obtained predictive samples from the model under consideration using each simulated posterior sample. The size of predictive samples is same as that of observed data.

The MCMC results of the posterior mean, median, mode of $\mathrm{X}$ two smallest $\left(X_{(1)}\right.$ and $\left.X_{(2)}\right)$ and two largest $\left(X_{(58)}\right.$ and $\left.X_{(59)}\right)$ are displayed in Table 5.3.

\begin{tabular}{lcccc}
\hline & Observed & Mode & Mean & Median \\
\hline $\mathrm{X}_{(1)}$ & 2.997 & 2.978 & 2.917 & 3.325 \\
$\mathrm{X}_{(2)}$ & 4.137 & 3.868 & 3.816 & 4.144 \\
$\mathrm{X}_{(58)}$ & 10.491 & 10.277 & 10.362 & 10.54 \\
$\mathrm{X}_{(59)}$ & 11.038 & 11.381 & 11.469 & 11.65 \\
\hline
\end{tabular}

Density estimates based on replicated future data sets are Table 5.3: Posterior characteristics shown in Figures 5.14 and 5.15. Figure 5.15 represents the estimates corresponding to largest two predictive observations, whereas the same for smallest two observations is shown in Figure 5.14. The corresponding observed values are also shown by means of vertical lines.
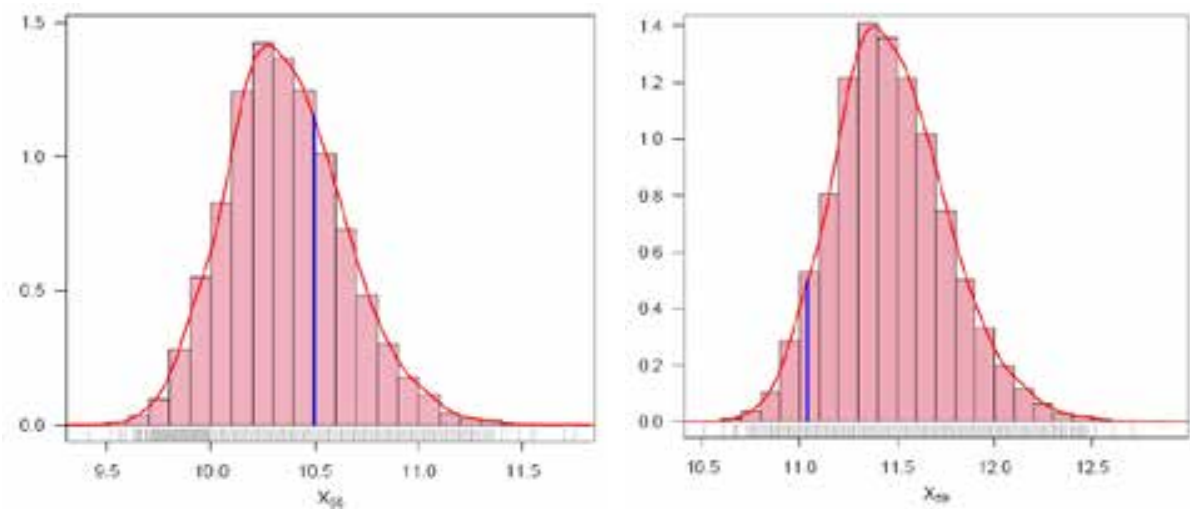

Fig 5.15 Density estimates of the two largest order future observations, vertical lines represent corresponding observed values 
In fact, we have predicated the entire data set. Figure 5.16 represents the Q-Q plot of predicted quantiles vs. observed quantiles. We, therefore, conclude that the Perks II model is compatible with the given data set.

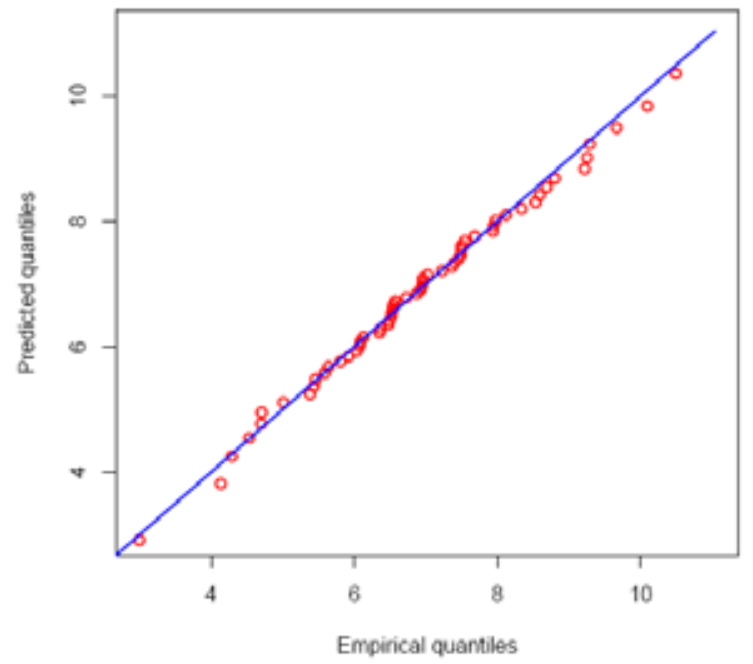

Fig. 5.16 Q-Q plot of predictive quantiles versus empirical quantiles

\subsection{Conclusion}

We have discussed the likelihood based inferential procedures (Classical as well as Bayesian) for Perks-II distribution.. We have developed tools for empirical modeling, e.g., model analysis, model validation and estimation of parameters. These tools are developed in R language and environment. The ML Estimates and their asymptotic confidence intervals of the parameters have been obtained. The procedures are developed to perform full Bayesian analysis of the Perks-II distributions using Markov Chain Monte Carlo (MCMC) simulation method in OpenBUGS, established software for Bayesian analysis using Markov Chain Monte Carlo (MCMC) methods. We have obtained the Bayes estimates of the parameters, hazard and reliability functions, and their probability intervals are also presented. We have also discussed the issue of model compatibility for the given data set. We have seen that Perks-II distributions is suitable for modelling the reliability data. 


\section{References}

Chaudhary, A.K \& Kumar,V (2013).A Bayesian Analysis of Perks-I distribution via Markov Chain Monte Carlo simulation,Nepal Journal of Science and Technology,14(1),153-166

Chen, M. H. \& Shao, Q. M. (1999). Monte Carlo estimation of Bayesian credible intervals and HPD intervals, Journal of Computational and Graphical Statistics. 8(1), 69-92.

Gelman, A. (2003). A Bayesian Formulation of Exploratory Data Analysis and Goodness-of-fit Testing, International Statistical Review, 71(2), 369-382.

Gelman, A., Carlin, J., Stern, H., \& Rubin, D. (2004). Bayesian Data Analysis, Second Edition, London, Chapman \& Hall.

Gupta, A., Mukherjee, B. \& Upadhyay, S.K. (2008). A Bayes study using Markov Chain Monte Carlo simulation, Reliability Engineering \& System Safety, 93, 1434-1443.

Haberman, S. \& Renshaw, A. (2011). A comparative study of parametric mortality projection models, Insur. Math. Econ., 48, 35-55.

Kumar, V. (2010). Bayesian analysis of exponential extension model, J. Nat. Acad. Math., Vol. 24, 109-128.

Lange, K. (1999). Numerical Analysis for Statisticians. Springer, New York, NY.

Lawless, J. F. (2003). Statistical Models and Methods for Lifetime Data, 2nd ed., John Wiley and Sons, New Jersey.

Lunn, D.J., Jackson, C., Best, N., Andrew, A., \& Spiegelhalter, D. (2013). The BUGS Book :A Practical Introduction to Bayesian Analysis, Chapman \& Hall/CRC, London, UK.

Marshall, A. W., \& Olkin, I.(2007). Life Distributions: Structure of Nonparametric, Semiparametric, and Parametric Families, Springer, New York.

Nadarajah, S. \& Bakar, S. A. A.(2012). ActuDistns : A new R package for actuarial survival models, http://cran.r-project.org/web/packages/ActuDistns/index.html.

Perks, W. (1932). On some experiments in the graduation of mortality statistics. Journal of the Institute of Actuaries, 43, 12-57.

R Development Core Team (2013). R: A language and environment for statistical computing. R Foundation for Statistical Computing, Vienna, Austria. ISBN 3-900051-07-0, URL http:// www.R-project.org.

Richards, S. J. (2012). A handbook of parametric survival models for actuarial use, Scandinavian Actuarial Journal, 1-25.

Richards, S.J. (2008). Applying survival models to pensioner mortality data, Br Actuar J., 14, 257303

Thomas,A.(2010). Open BUGS Developer Manual, version 3.1.2, http://www.openbugs.info/

Thomas, A., O'Hara, B., Ligges, U. \& Sturtz, S. (2006). Making BUGS Open, R News, 6, 12-17, URL http://mathstat.helsinki.fi/openbugs/.Yee, T. W. (2012). The VGAM Package, R News, 8, 2839. 